\title{
Determinación de los potenciales evocados visuales en el personal administrativo con hipertensión de la Universidad de Panamá
}

\author{
Obando, Xavier \\ Laboratorio de Neurofisiología, Facultad de Medicina, Universidad de Panamá \\ Panamá, Panamá \\ obandoup@gmail.com \\ Calvo, Oris \\ Laboratorio de Neurofisiología, Facultad de Medicina, Universidad de Panamá \\ Panamá, Panamá \\ oriscalvo20@gmail.com
}

\section{Abstract}

Visual evoked potentials (VEPs) are bioelectric signals generated in the striated and extrastriated cortex when the retina is stimulated with light or through changes in patterns projected by a screen. Currently VEPs are used mainly as a valuable tool to determine hidden lesions of the visual pathway. Some recent studies carried out in Asian countries have shown findings that suggest that hypertension affects the neural nerve conduction of the visual pathway and that it can be detected in advance through the use of visual evoked potentials. In Panama there are no studies carried out to support the information provided by the theory, so this study is intended to determine the PEVs to provide important information on the values obtained in relation to their state of health that may alter the visual nervous pathway, being hypertension the main reason for study. The PEVs were carried out in the Neurophysiology Laboratory of the School of Medicine, University of Panama, with the Neurowerk - SIGMA team. As preliminary results, no variation was observed in the latencies obtained between control and hypertensive subjects. Regarding the amplitude, a slight decrease was observed in hypertensive subjects. The information obtained in this research will be used to provide data on the values of the visual evoked potentials applicable to the Panamanian population; in addition to providing adequate treatment and follow-up to people with hypertension to avoid injuries to the visual pathway. 
Keywords: Visual evoked potentials, hypertension, visual pathway, bioelectric signals, visual pathology.

\section{Resumen}

Los potenciales evocados visuales (PEV), son señales bioeléctricas generadas en la corteza estriada y extraestriada cuando la retina es estimulada con luz o mediante cambios de patrones proyectados por una pantalla. En la actualidad los PEV son utilizados principalmente como una herramienta valiosa para determinar lesiones ocultas de la vía visual. Algunos estudios recientes realizados en países asiáticos han mostrado hallazgos que sugieren que la hipertensión afecta la conducción nerviosa de la vía visual y que puede ser detectada con anticipación por medio del empleo de los potenciales evocados visuales. En Panamá no hay estudios realizados que sustenten la información que nos proporciona la teoría, por lo que se pretende con este estudio determinar los PEV para proporcionar información importante sobre los valores obtenidos con relación a su estado de salud que puedan alterar la vía nerviosa visual, siendo la hipertensión el principal motivo de estudio. La realización de los PEV se realizó en el Laboratorio de Neurofisiología de la Facultad de Medicina, Universidad de Panamá, con el equipo Neurowerk - SIGMA. Resultados preliminares muestran que no hubo variación de las latencias obtenidas entre los sujetos controles e hipertensos. En cuanto a la amplitud si se observó una ligera disminución en los sujetos hipertensos. La información obtenida en esta investigación se utilizará para proporcionar datos de los valores de los potenciales evocados visuales a aplicables a la población panameña; además de proporcionar un tratamiento y seguimiento adecuado a las personas con hipertensión para evitar las lesiones en la vía visual.

Palabras claves: Potenciales evocados visuales, hipertensión, vía visual, señales bioeléctricas, patología visual.

\section{INTRODUCCIÓN}

El estudio de los potenciales evocados visuales es de utilidad clínica en pacientes con Hipertensión arterial, ya que permite evaluar los posibles daños en la vía visual que puede ocasionar esta patología (Rodríguez et al. 2008).

La hipertensión es un problema de salud pública, siendo una de las enfermedades más comunes en nuestra sociedad (Nigam et al. 2016). Las personas con retinopatía hipertensiva permanecen durante muchos años sin síntomas, porque el daño que produce 
la hipertensión en los vasos de la retina es muy lento, causando inicialmente isquemia lo cual puede causar daños de las células ganglionares de la retina o desmielinización de las fibras nerviosas que llevan la información sensorial visual a los centros superiores, antes de que se presenten sintomatología muy avanzadas como visión borrosa, disminución de la agudeza visual, e incluso, pérdida de la visión (Capote 2019).

En Panamá la hipertensión representa el séptimo lugar de las causas de muerte, con una tasa de mortalidad del $20.1 \%$ en total de la población general. Siendo mayor la tasa de mortalidad en hombres con un (21.1\%), que con las mujeres (19.0\%) siendo también uno de las principales enfermedades no transmisibles que afectan a nuestra población (Ministerio de Salud Panamá 2016; INEC - Contraloria de Panamá 2018).

Se han realizado muy pocos estudios para evaluar las anomalías de las vías visuales en hipertensión, por lo que en el presente estudio se está realizando para evaluar los cambios de los PEV en una población hipertensa.

\section{MÉTODO}

\section{A. DOCUMENTACIÓN Y PRUEBAS PREVIAS}

Previo a la realización de la prueba de los PEV, se le explica al sujeto la técnica de registro, riesgos y complicaciones; información que está detallada en el consentimiento informado aprobado por el comité de bioética de la Universidad de Panamá. Se realiza una historia clínica donde se establece la condición de hipertensión del sujeto y los criterios de exclusión. Se procede a determina la presión arterial del sujeto por el método auscultatorio.

\section{B. EVALUACIÓN DE LOS POTENCIALES EVOCADOS VISUALES}

Para el registro de electrofisiológico de los PEV, se utilizó el equipo Neurowerk - SIGMA. Se establecieron los siguientes parámetros de registro y estimulación:

- Se realizó el procedimiento utilizando lentes correctivos si es necesario.

- Se registraron monocularmente los PEV

- El sujeto se colocó a una distancia de 1,60 metros de la pantalla.

- El electrodo de registro se colocó en la región occipital, a $5 \mathrm{~cm}$ por arriba del inión.

- Tamaño del tablero de ajedrez 60 min (1 grado).

- La luminancia de la pantalla $100 \mathrm{~cd} / \mathrm{m} 2$

- Sensibilidad 4,0 uV/DIV

- Base de tiempo 20ms/DIV

- Ciclos 100/100, por cada medición.

- Tiempo de estímulo: repetitivo

- Frecuencia de estímulo: $1,7 \mathrm{~Hz}$

- Filtro Notch: Encendido 


\section{RESULTADOS}

Se realizo el estudio para comparar los cambios en los PEV en sujetos hipertensos con respecto a sujetos control sanos. Se evaluó la correlación de las respuestas observadas en la prueba en cuanto a las Ondas N75, P100 y N140; y la amplitud de la Onda P100 en un patrón de cambio de 60 minutos.

La figura 1, muestra el registro de los PEV realizado en un sujeto control. Los resultados de los estudios realizados en sujetos normales evalúan principalmente la presencia de la onda P100, la cual debe mantenerse en valores normales dentro de 115 a 120 ms, mientras que la amplitud no debe presentar una alteración o disminución ya que puede indicar alteraciones en la vía nerviosa visual (Rubin and Daube 2016).

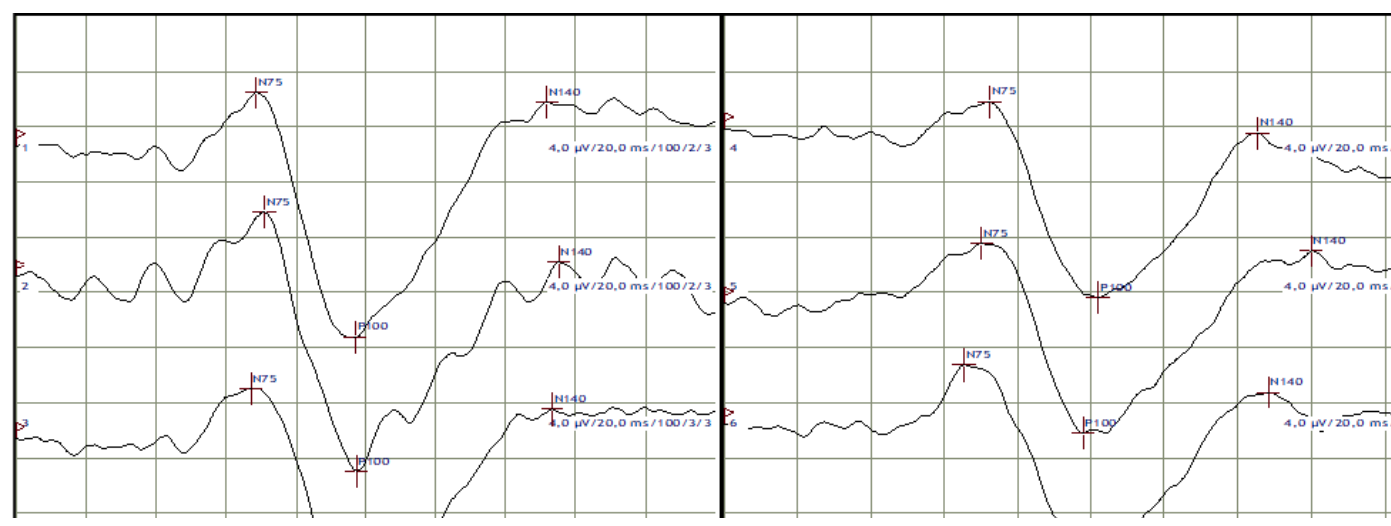

Fig. 1. Registro de los Potenciales Evocados Visuales de un sujeto control.

En la figura 2, se puede observar un registro de los PEV realizado un sujeto hipertenso, el cual presenta una morfología alterada y una amplitud disminuida. Las curvas parecidas en algunos estudios han demostrado que el aumento de la presión arterial puede causar aumento de la latencia de la onda P100 y una disminución de su amplitud (Balakrishnan and Natarajan 2018; Anitha Achuthan and Girija 2015).

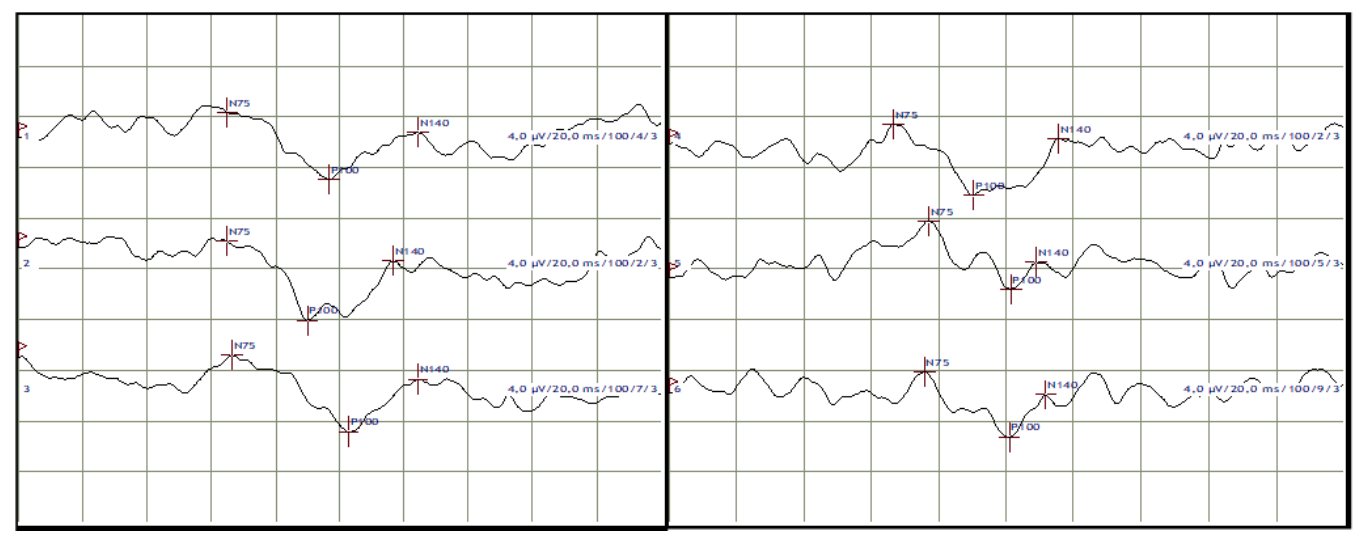

Fig. 2. Registro de los Potenciales Evocados Visuales de un sujeto hipertenso 
Los resultados preliminares mostraron que los valores de la latencia P100 entre los controles y los hipertensos no mostraron diferencia con respecto al rango establecido por los valores normativos de la Clínica Mayo, (por debajo de 115 - $120 \mathrm{mseg}$ ). Los valores de las latencias N75 y N140, en ambos grupos muestran que se encuentran dentro de los rangos establecidos, por debajo de $75 \mathrm{mseg}$ y $140 \mathrm{mseg}$ respectivamente (Figura 3 ). En cuanto al tamaño de la amplitud de la onda P100, en los sujetos hipertensos mostró una ligera disminución en comparación con los pacientes controles, que de acuerdo con la literatura se correlaciona con un aumento de la presión arterial. La morfología de la onda P100 mostró igualmente cambios asociados a la Hipertensión Arterial (Figura 4). Los resultados presentados son descriptivos y preliminares porque aún no se ha completado el registro del número de casos de la investigación.

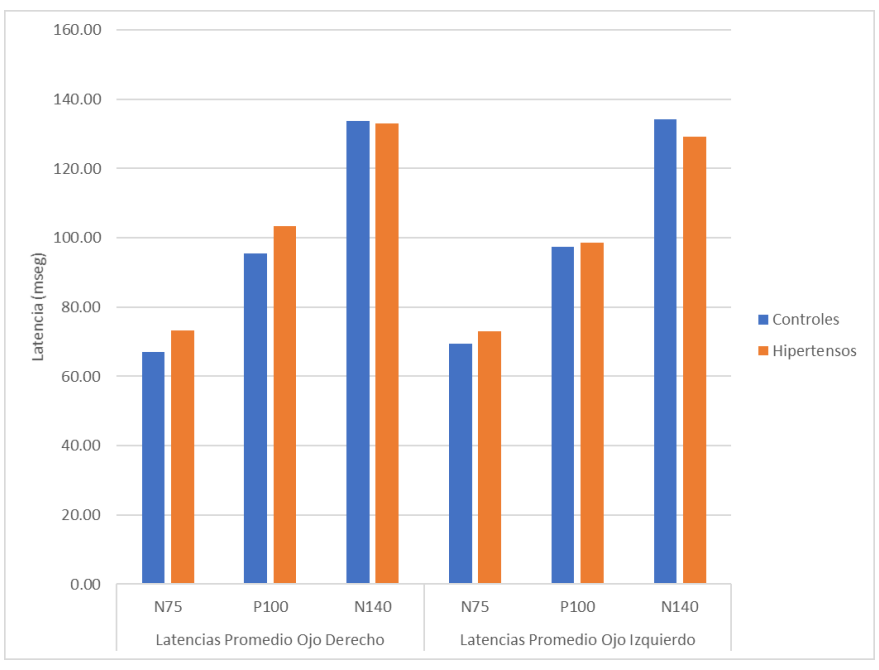

Figura 3. Latencia de las Ondas N75, P100, N140 en controles e Hipertensos

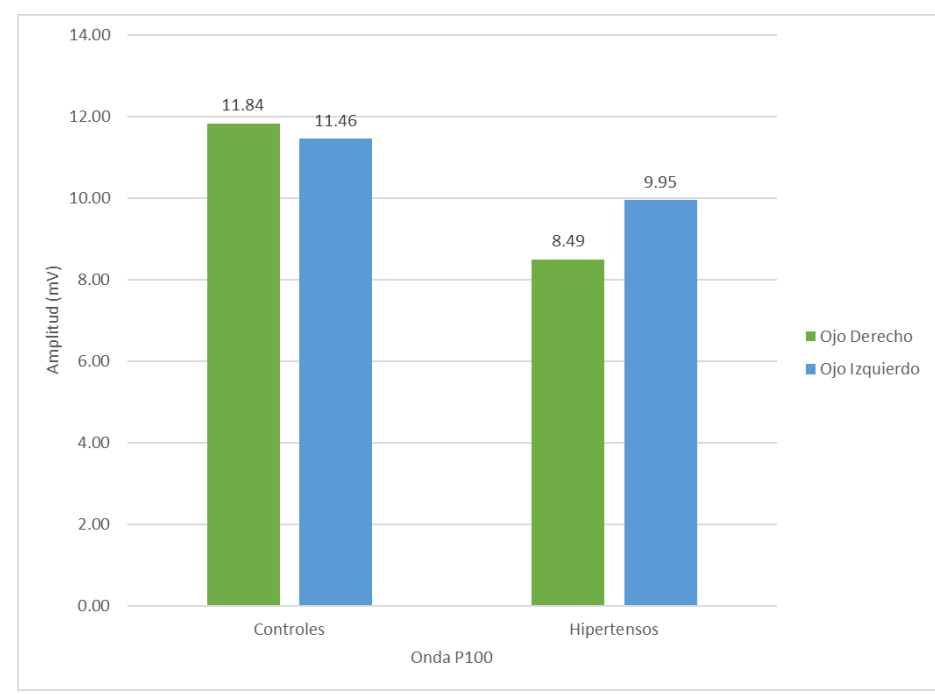


Figura 4. Amplitud de las Ondas P100 en Controles e Hipertensos

\section{CONCLUSIONES}

Los resultados presentados en este estudio son descriptivos y preliminares debido a que aún no se ha completado el registro del número de casos de la investigación por motivos de pandemia.

En cuanto a la morfología de los registros de los PEV, se pudo observar una alteración en los sujetos hipertensos en comparación de los sujetos controles, que mostraron una morfología en sus ondas constantes. Se pudo observar que la latencia de las ondas tanto en sujetos control como en sujetos hipertensos no mostraron una variación en cuanto a sus resultados ya que todos se mantuvieron dentro de los valores normales, mientras que en la amplitud de las ondas se pudo observar una ligera disminución en sujetos con hipertensión.

Los cambios de los potenciales evocados visuales ocurren en sujetos hipertensos antes del desarrollo de la retinopatía hipertensiva. Por lo tanto, la medición de los PEV se puede utilizar como un examen de rutina para el seguimiento de los pacientes hipertensos y el diagnóstico temprano de la retinopatía hipertensiva que trae como consecuencia alteraciones en la vía visual. El registro de los PEV también serviría para realizar cambios y/o ajustes en las dosis de los antihipertensivos para el mejor control de los valores de la presión arterial y así evitar los daños en la vía visual.

\section{Referencias}

[1] N. Rodríguez, A. Zurutuza, A. Nelson, and M. Rodríguez. “Manifestaciones Oftalmológicas de La Hipertensión Arterial Ophthalmological Manifestations of Arterial Hypertension Correspondencia :" Anales del Sistema Sanitario de Navarra 2008; 31 (Supl. 3): 13-22.

[2] J. Nigam, S. Ajit, V.Virendra, J. Vikas, G. Jain, and A.Shrivastava. "Study of ' Visual Evoked Potential ' Variations in Primary Hypertensive". International Journal of Heath Science and Research, 6 (September 2016): 164-68.

[3] M. Capote. "La Hipertensión Provoca Problemas En La Vista a Un 11\% de Los Que La Padecen." E. Press. Europa Press. 2019. https://www.infosalus.com/asistencia/noticia-hipertension-provocaproblemas-oculares-11-padecen-20190517105614.html.

[4] Ministerio de Salud Panamá. 2016. "Indicadores Básicos de Panamá 2016." Ministerio de Salud, 1-24. http://www.minsa.gob.pa/sites/default/files/publicacion-general/indicadores_basicos_de_ salud_2016.pdf.

[5] INEC - Contraloria de Panamá. "Defunciones y Tasa de Mortalidad de Las Principales Causas de Muerte En La República de Panamá, Por Sexo, Según Causa: Año 2018." Vol. 6. https://www.inec. gob.pa/archivos/P0579518620191205111309Cuadro 11.pdf.

[6] D. Rubin and J. Daube. Clinical Neurophysiology. Oxford. 4ta Edition. 2016.

[7] A. Balakrishnan and N. Nirmala. "A Comparative Study on Visual Evoked Potential in Normotensive and Hypertensive Individuals." National Journal of Physiology, Pharmacy and Pharmacology 2018. 8 
(10): 1437-40. https://doi.org/10.5455/njppp.2018.8.0620723072018.

[8] A. Achuthan and S. Girija. "Pattern Visual Evoked Potential in Newly Diagnosed Hypertensive Individuals." International Journal of Research in Medical Sciences. 2015.3 (12): 3614-19.

\section{Autorización y Licencia CC}

Los autores autorizan a APANAC XVIII a publicar el artículo en las actas de la conferencia en Acceso Abierto (Open Access) en diversos formatos digitales (PDF, HTML, EPUB) e integrarlos en diversas plataformas online como repositorios y bases de datos bajo la licencia CC:

Attribution-NonCommercial-ShareAlike 4.0 International (CC BY-NC-SA 4.0) https://creativecommons.org/ licenses/by-nc-sa/4.0/.

Ni APANAC XVIII ni los editores son responsables ni del contenido ni de las implicaciones de lo expresado en el artículo. 